\title{
PROSPECTIVE OVERRULING: BILANCIO DI UNA VICENDA ${ }^{1}$
}

\section{PROSPECTIVE OVERRULING: BALANCE OF AN AFFAIR}

Remo Caponi

Professor da Università degli Studi di Firenze, Italia.

E-mail: remo.caponi@gmail.com

RIASSUNTO: L'articolo affronta il tema del superamento futuro, come tecnica per modificare i precedenti, e limita l'efficacia retroattiva delle decisioni giudiziarie.

PAROLE CHIAVE: precedenti; processo giusto; giusto processo; fonti della legge; efficacia procesuale.

ABSTRACT: The article addresses the topic of prospective overruling, as a technique for modifying precedents, and limits the retroactive effectiveness of judicial decisions.

KEY WORDS: precedents; right process; due right process; sources of the law; procesual effectiveness.

SOMMARIO: 1. Il problema. - 2. Finzione perfetta. - 3. Modificare la teoria, non mistificare la realtà. - 4. Efficacia retrospettiva dell'interpretazione giudiziale. - 5. Precedente giudiziale come fonte del diritto. - 6. Dogmi giuridici come "polmoni aperti sull'esperienza". - 7. Fatti "tiranni". 8. Giusto processo. - 9. Applicazione diretta del giusto processo.

\section{Problema}

L'individuazione dei limiti della retroattività del mutamento di giurisprudenza (prospective overruling) è un problema classico nei sistemi di common law, e - almeno dagli inizi degli anni settanta del secolo XX - anche nei sistemi di civil law.

\footnotetext{
${ }^{1}$ Artigo recebido em 30/05/2018, sob dispensa de revisão.
} 
La situazione creata da Cass. 9 settembre 2010, n. $19246^{2}$ ha sollevato il problema anche nell'esperienza giuridica italiana, risvegliandola dal "sonno dogmatico" che la cullava nell'idea di una retroattività costante, illimitata, inevitabile delle pronunce giudiziali.

Alla stregua del diritto vivente fino a questa sentenza, la riduzione alla metà del termine di costituzione dell'opponente a decreto ingiuntivo, ai sensi dell'art. 645, comma 2, c.p.c., era un effetto conseguente al fatto che l'opponente avesse assegnato all'opposto un termine a comparire inferiore a quello legale e non già alla semplice proposizione dell'opposizione. Gli opponenti che avevano assegnato all'opposto un termine di comparizione pari o superiore a quello legale e che si erano costituiti in giudizio entro dieci giorni dalla notificazione dell'opposizione potevano confidare sulla procedibilità dell'opposizione.

Con Cass. n. 19246 del 2010, questo affidamento era venuto meno: le costituzioni in giudizio dell'opponente successive al quinto giorno dalla notificazione dell'opposizione, tempestive secondo il diritto vivente al tempo in cui sono avvenute, avrebbero potuto essere qualificate come tardive. Nei giudizi ancora in corso, l'opposizione avrebbe potuto essere dichiarata improcedibile e il decreto ingiuntivo avrebbe potuto consolidarsi come immutabile.

\section{Finzione perfetta}

Al momento del risveglio dal sonno dogmatico, di fronte ad una situazione (quella originata appunto da Cass. n. 19246 del 2010) che si ribellava ad essere assoggettata al principio della retroattività, non si è modificato il principio, ma si è ricostruita la realtà in modo diverso dal vero, facendo ricorso alla rimessione in termini.

L'impiego di questo rimedio è frutto di una finzione perfetta, sotto un duplice profilo. In primo luogo, essa rispetta integralmente l'idea della retroattività del mutamento di giurisprudenza, fingendo che la parte sia incorsa in un errore o in un impedimento. In secondo luogo, essa perviene ad un risultato integralmente equo, riconoscendo la scusabilità dell'errore, la non imputabilità dell'impedimento. Qualche difficoltà dal punto di vista tecnico può sorgere solo perché occorrerebbe sempre l'istanza di parte.

Si parva licet, si è riprodotta una situazione analoga a quella anteriore al riconoscimento che, nella responsabilità aquiliana, esistono criteri di imputazione oggettiva della responsabilità alternativi rispetto al criterio di imputazione della colpa. In quella vicenda ormai superata, si

\footnotetext{
${ }^{2}$ In Foro it. 2010, I, 3014.
} 
Revista Eletrônica de Direito Processual - REDP.

Rio de Janeiro. Ano 12. Volume 19. Número 2. Maio a Agosto de 2018

Periódico Quadrimestral da Pós-Graduação Stricto Sensu em Direito Processual da UERJ

Patrono: José Carlos Barbosa Moreira (in mem.). ISSN 1982-7636. pp. 173-183

www.redp.uerj.br

fingevano colpe inesistenti per rimanere fedeli a tutti i costi al principio della colpa. Nella vicenda attuale, si fingono errori e impedimenti inesistenti per rimanere fedeli a tutti i costi al principio di retroattività del mutamento di giurisprudenza.

\section{Modificare la teoria, non mistificare la realtà}

Se si rimane sul piano gnoseologico, il contrasto tra teoria e realtà non si compone in modo migliore se si modifica la realtà (cioè se si finge una realtà inesistente), ma se si modifica la teoria. Perciò si lascia particolarmente apprezzare Cass. sez. un. 11 luglio 2011, n. $15144^{3}$. Pronunciata su fattispecie analoga quella sottesa a Cass. n. 19246 del 2010, questa sentenza non ricorre a finzioni, ma inquadra la realtà e la qualifica giuridicamente nei termini corretti.

Essa esamina in modo autoriflessivo uno dopo l'altro gli aspetti fondamentali relativi al mutamento di giurisprudenza consolidata sull'interpretazione di una norma processuale, laddove il nuovo orientamento, se applicato alle situazioni processuali anteriori, precluderebbe ad una parte di agire o di proseguire il giudizio, mentre secondo il precedente orientamento, in essere al momento del verificarsi della predetta situazione processuale, la parte potrebbe agire o proseguire il giudizio.

L'impatto pratico di questa sentenza è determinato da due affermazioni concatenate. Primo: “sono proprio le peculiari connotazioni dell'overruling che, per la loro eccezionalità [..], giustificano una scissione tra il fatto (il comportamento della parte risultante ex post non conforme alla corretta regola del processo) e l'effetto, di preclusione, che dovrebbe derivarne". Secondo: "nel caso [..] in cui venga, come nella specie, in rilievo un problema di tempestività dell'atto (sussistente in base alla giurisprudenza overruled, ma venuta meno in conseguenza del successivo mutamento di esegesi della regola di riferimento), il valore del giusto processo può trovare diretta attuazione attraverso l'esclusa operatività, come detto, della preclusione derivante dall'overruling nei confronti della parte che abbia confidato nella consolidata precedente interpretazione della regola stessa".

Le sezioni unite della Corte di cassazione escludono, in questo caso, la normale efficacia retroattiva del mutamento di giurisprudenza. Coerentemente, non ritengono necessario che sia esperita la rimessione in termini e distinguono così espressamente la propria soluzione da quella

\footnotetext{
${ }^{3}$ In Foro it., 2011, I, 2254.
} 
sostenuta da Cass. 16 giugno 2010, n. $14627^{4}$. Tuttavia quest'ultima pronuncia ha avuto il merito di sollevare il problema, di affrontarlo in modo approfondito, e di conseguire un risultato equo, in un momento in cuisi tendeva a ritenere la rimessione in termini il rimedio tecnicamente corretto, prima che la vicenda originata da Cass. n. 19246 del 2010 inducesse almeno qualcuno a sostenere un'altra tesi.

Aggiungendo una propria, notevole forza argomentativa, Cass. n. 15144 del 2011 accoglie quest'altra tesi.

\section{Efficacia retrospettiva dell'interpretazione giudiziale}

Le due fattispecie sottese a Cass. n. 19246 del 2010 e a Cass. n. 15144 del 2011 sono accomunate nel loro tratto saliente: il mutamento di giurisprudenza mette in gioco il diritto di una parte di agire o di proseguire il giudizio. Nel proseguimento, tengo presente per comodità solo la vicenda dei termini di costituzione nel giudizio di opposizione al decreto ingiuntivo. Riprendo la tesi, accolta da Cass n. 15144 del 2011, nella formulazione che le avevo dato in un incontro di studi presso la Corte di Cassazione nel $2011^{5}$.

Punto di partenza è che l'interpretazione giudiziale del diritto ha carattere dichiarativo e quindi in via di principio efficacia retroattiva (retrospettiva). Se non avessi un poco di timore nell'appoggiarmi a giustificazioni giuridiche che si appellano alla natura delle cose, direi che si tratta di un dogma tratto dal modo di essere della pronuncia giudiziale.

Questa efficacia retroattiva ha carattere vincolante con riferimento alla fattispecie decisa (tranne che si tratti di un obiter dictum, che per definizione non riguarda quest'ultima) e carattere persuasivo con riferimento alle altre fattispecie analoghe. Capita a volte di sentir dire che "un precedente esplica efficacia anche se non persuade", ma questa affermazione non è condivisibile. Se il precedente non persuade, il giudice chiamato successivamente a decidere una controversia analoga può motivatamente distaccarsene. Questa strada è stata sicuramente seguita da molti giudici all'indomani di Cass. n. 19246 del 2010 (nel senso che non l'hanno applicata alle fattispecie anteriori). Sennonché, la normale tendenza dei giudici di merito a mantenere fermi i giudizi della Corte di cassazione e il dogma della assoluta retroattività del mutamento di

\footnotetext{
${ }^{4}$ In Foro it., 2010, I, 3050.

${ }^{5} \mathrm{Si}$ tratta dell'Incontro di studi su Norme processuali e mutamenti di giurisprudenza, organizzato dalla Formazione decentrata del Consiglio Superiore della Magistratura presso la Corte di cassazione, 24 maggio 2011, citato nell'asterisco iniziale.
} 
giurisprudenza hanno spronato la Corte di cassazione ad intervenire di nuovo, alla prima occasione utile.

\section{Precedente giudiziale come fonte del diritto}

Merita un cenno il problema dell'inserimento dell'efficacia persuasiva del precedente giurisprudenziale, specialmente di quello delle Corti supreme, tra le fonti del diritto. Si tratta di un problema teorico che non incide strettamente sul nostro tema, ma non voglio evitare di prendere posizione.

Nel passaggio dall'assolutismo giuridico, in cui la legge statale era tutto il diritto, ad un forte assetto pluralistico, con il moltiplicarsi e l'intrecciarsi dei piani di legalità (regionale, statale, costituzionale, sovranazionale, internazionale), sono venute meno le ragioni storiche che hanno sostenuto la negazione del carattere di fonte del diritto delle pronunce giudiziali. Passerà ancora del tempo prima che se rendano conto tutti, ma la direzione di marcia è segnata. Certamente, questo riconoscimento deve rispettare le caratteristiche proprie delle pronunce giudiziali e distinguere le ipotesi in cui la sentenza giudiziaria è fonte di norme interpretative, oppure di norme integrative. A proposito di queste ultime, la terminologia tedesca conosce un termine pregnante: Rechtsfortbildung: letteralmente, ulteriore costruzione, perfezionamento del diritto. Ciò accade se le pronunce dei giudici colmano lacune. Ciò è inteso in accezione ampia, che comprende anche la rimodulazione di dogmi giuridici, come specie di risoluzione di una lacuna valutativa. Vi rientra anche il caso della modificazione del dogma della retroattività illimitata della pronuncia giudiziale.

\section{Dogmi giuridici come "polmoni aperti sull'esperienza"}

I dogmi giuridici sono costruzioni intellettuali, frutto della cultura giuridica assistita dalla logica, ma non danno luogo ad imposizioni inevitabili e assolute. Essi sono disponibili ad essere rielaborati da quella stessa cultura che li ha creati. I dogmi giuridici sono aperti ad una ricostruzione evolutiva. Non sono mai assoluti, sono intrinsecamente relativi. Anche i dogmi giuridici imparano dalla vita, come dovrebbe imparare il legislatore, come dovremmo imparare tutti noi. Essi sono al servizio della vita. Non è la vita al servizio dei dogmi. 
Il meccanismo che consente ad una costruzione giuridica di imparare dalla vita è l'eterno rapporto tra regola ed eccezione. Questo rapporto assicura il progresso del diritto. Tra regola ed eccezione non vi è opposizione, ma vi è un intreccio vitale, "L'eccezione è più interessante del caso normale. Quest'ultimo non prova nulla, l'eccezione prova tutto; non solo essa conferma la regola: la regola stessa vive solo dell'eccezione" (C. Schmitt). Vuol dire che la regola si coglie nel suo pieno significato solo dal punto di vista dell'eccezione, e viceversa. Si deve diffidare delle regole che non conoscono eccezioni. A ben vedere, non sono più nemmeno regole: diventano necessità naturali.

\section{Fatti "tiranni"}

Nella vita si verificano a volte dei fatti "tiranni": sono quei fatti che, per il modo di essere e di verificarsi, reclamano quasi con violenza di essere trattati in modo diverso dalla regola cui dovrebbero essere sottoposti. Sono fatti che vanno quasi da soli alla ricerca della loro regola, conformando in una certa misura lo stesso parametro valutativo. La vicenda originata da Cass. $\mathrm{n}$. 19246 del 2010 appartiene al novero di questi fatti tiranni. Ne metto in fila i tratti:

a) mutamento di giurisprudenza per obiter dictum, mentre un'ampia interpretazione dell'art. 363 , comma 3 c.p.c. dovrebbe escludere questa eventualità;

b) mutamento di orientamento di giurisprudenza consolidato da oltre mezzo secolo;

c) mutamento di giurisprudenza in materia di interpretazione di una norma processuale, mentre non si dovrebbero cambiare le regole del processo quando esso è in corso;

d) cambiamento di giurisprudenza della corte di cassazione, cui l'ordinamento italiano assegna una funzione di guida interpretativa; 5

e) cambiamento di giurisprudenza che priva del diritto al processo chi si è affidato incolpevolmente al precedente orientamento. Tutti questi elementi (soprattutto l'ultimo, come già detto) delineano i contorni di un fatto eccezionale, che impone di essere trattato in modo diverso rispetto alla regola della retroattività del mutamento di giurisprudenza.

\section{Giusto processo}

Ovviamente un fatto, per quanto eccezionale, non diventa di per sé un valore. Occorre trovare una norma giuridica in grado di valorizzarlo. Nel nostro caso, occorre trovare una norma 
giuridica in grado di sottrarre la vicenda originata da Cass. n. 19246 del 2010 alla regola della retroattività del mutamento di giurisprudenza. Questa norma è il giusto processo. In particolare, l'aspetto della garanzia costituzionale che consiste nella tutela dell'affidamento incolpevole nella predeterminazione delle regole di svolgimento del processo (art. 111 Cost.).

Se il giusto processo è una norma giuridica (una sintesi di norme giuridiche) e se le norme giuridiche hanno la virtù di disciplinare direttamente i fatti della vita, non vi è dubbio che la garanzia del giusto processo impone di limitare, in questo caso, l'efficacia retroattiva del mutamento di giurisprudenza costante della Corte di cassazione sulla interpretazione dell'art. 645, comma 2 c.p.c., senza necessità di richiedere la rimessione in termini, poiché l'affidamento nel precedente orientamento di giurisprudenza sull'interpretazione dell'art. 645, comma 2 c.p.c. è in re ipsa incolpevole.

Laddove la "causa" è in re ipsa "non imputabile", la richiesta di rimessione in termini è superflua, come in ipotesi di tardivo rilievo di una questione rilevabile d'ufficio, o di ammissione di un mezzo di prova, da parte del giudice. Gli ultimi due esempi confermano che la soluzione accolta da Cass. n. 15144 del 2011, pur dettata da una vicenda eccezionale, si inserisce in una "rima obbligata" (à la Crisafulli) del sistema.

\section{Applicazione diretta del giusto processo}

Questa operazione è possibile, perché in questo caso non vi è una regola legislativa che si interpone tra principio costituzionale e fatto, impedendo al primo di disciplinare il secondo direttamente (indirettamente, in due modi, il principio costituzionale disciplina il fatto anche quando tra i due si frappone una regola legislativa: ispirando una interpretazione conforme della regola legislativa, oppure - se il contrasto della regola con il principio non si può risolvere con una interpretazione conforme - offrendo il criterio al sindacato di costituzionalità).

In altri termini, la vicenda di cui si tratta è radicalmente diversa da quella che ha (in modo non condivisibile) condotto la Corte di cassazione a sacrificare l'art. 37 c.p.c. in vista della ragionevole durata del processo (Cass. sez. un. 9 ottobre 2008, n. $24883^{6}$ ). In questa vicenda ci troviamo invece a rimodulare l'applicazione di un dogma (quello della retroattività della interpretazione giudiziale del diritto) con gli stessi strumenti della ragion pratica con cui esso è stato elaborato.

\footnotetext{
${ }^{6}$ In Foro it., 2009, I, 806.
} 


\section{Formazione giudiziale e formazione legislativa del diritto}

Ho ripetuto frequentemente che la limitazione della normale retroattività dell'interpretazione giudiziale del diritto ha luogo in questo caso concreto, è collegata a questa vicenda concreta: il mutamento di giurisprudenza mette in gioco il diritto di una parte di agire o di proseguire il giudizio.

Qual è il sottofondo della insistenza nel richiamo alla vicenda concreta? Si è già detto che le pronunce dei giudici, e specialmente quelle delle corti supreme, possono costituire dei tasselli normativi che integrano il sistema giuridico.

Tuttavia (questo è il punto) la formazione giudiziale del diritto è qualitativamente diversa da quella legislativa (ci si riferisce ad un tema diverso dalla distinzione tra efficacia vincolante ed efficacia persuasiva).

La brevità di questo contributo mi consente una semplificazione brutale: mentre la legge è una previsione normativa (se $\mathrm{x}$, allora $\mathrm{y}$ ) e nasce (dovrebbe nascere) in esecuzione di un disegno politico, la sentenza è un accertamento normativo (poiché x, allora y), che nasce letteralmente "per caso" e "per processo" (giurisdizionale), perché si è già verificato un caso, una controversia che richiede di essere risolta.

La portata della norma legislativa (in particolare di quella che abbia struttura di regola), la sua capacità di disciplinare i futuri contegni umani è individuata attraverso una comparazione della fattispecie concreta da disciplinare sia con la fattispecie legale, sia anche - soprattutto - con la ratio legis, che sorregge la previsione di quest'ultima. La ratio legis esiste prima del fatto da disciplinare: la regola legislativa è appunto una previsione normativa. La ratio legis ha frequentemente, per non dire sempre, una proiezione normativa che eccede la descrizione legale della fattispecie: i vecchi brocardi dell'antica sapienza legis plus dixit quam voluit, lex minus dixit quam voluit lo confermano.

La ratio decidendi (in particolare quando il parametro sia un principio) ha natura ed attitudine normativa diverse dalla ratio legis. La ratio decidendi è saldamente agganciata a quel fatto che si è già verificato nel passato e ha dato origine alla pronuncia giudiziale, un fatto che proietta permanentemente la propria ombra sul dictum giudiziale, con tutta la maggiore forza che, rispetto alla fattispecie legale astratta, gli deriva sia dall'essersi già verificato nella realtà, sia dal non dover temere la concorrenza della ratio legis. Da un lato, questo tratto rivela la debolezza della portata normativa della pronuncia giudiziale rispetto alla legge, che in apicibus discende 
dal principio della domanda, dal carattere necessariamente reattivo dell'intervento giurisdizionale (e infatti la Corte di cassazione ha dovuto attendere il riproporsi di una fattispecie analoga a quella sottesa a Cass. n. 19246 del 2010 per intervenire di nuovo). Dall'altro lato, questo tratto rivela la forza degli orientamenti giurisprudenziali: la loro capacità di reagire con circospezione, passo dopo passo, fatto dopo fatto, ai mutamenti della realtà.

Il problema consiste nell'approfondire come operi questa evoluzione giurisprudenziale sollecitata dai nuovi fatti da giudicare. L'individuazione dei fatti rilevanti per la decisione del caso è un'opera di selezione e di costruzione, di tipizzazione in una fattispecie astratta (analoga alla fattispecie della norma giuridica, laddove quest'ultima rivesta la struttura della regola, G. Gorla) dei fatti che il giudice trova dinanzi a sé, o meglio dei racconti che ne fanno le parti. È questa fattispecie ricostruita che è descritta nella ratio decidendi e trova espressione nella massima giurisprudenziale che viene elaborata poi dagli uffici studio delle corti e dalle redazioni delle riviste giuridiche. Al momento della decisione, anche la norma giuridica espressa in forma di principio si concretizza evidentemente in una regola. Ma le regole giurisprudenziali, le rationes decidendi, si applicano a quei casi della vita che giungono dinanzi al giudice, non alla fattispecie astratta che ne viene poi desunta. Ne segue che la ricostruzione degli orientamenti della giurisprudenza non si può compiere se non attraverso la lettura attenta e integrale del testo delle sentenze, e non delle massime che se ne estraggono. La prima rilettura attenta e integrale del testo integrale delle sentenze è quella che fa la corte stessa, con riferimento ai propri precedenti, per saggiare se vi sono margini per una conferma, per un distinguishing, se non per una modifica del proprio orientamento. La valutazione comparativa ha per oggetto le due narrazioni complete dei fatti della vita: la precedente e l'attuale. Si tratta di un lavoro lungo, che è da compiere senza risparmio di energie.

Di questa circospezione ha dato grande prova Cass. n. 15144 del 2011. Il risultato conseguito lascia aperta la possibilità di reazione flessibile della giurisprudenza. Ad esempio, può accadere che in vicende diverse da quella originata da Cass. n. 19246 del 2010 si presenti la necessità di accertare in concreto il carattere incolpevole dell'affidamento nel precedente orientamento di giurisprudenza, anche in relazione al momento in cui l'affidamento cessa di essere incolpevole ${ }^{7}$.

\section{Appendice bibliografica}

\footnotetext{
${ }^{7}$ Vedi per esempio Cass. 7 febbraio 2011, n. 3030, in Foro it., 2011, I, 1080.
} 
Le osservazioni che precedono sono frutto di studi, riflessioni e dialoghi su questi temi (e su temi collegati), ormai stratificati nel corso di un quarto di secolo. Ho avvertito difficoltà a collegare tali studi a punti specifici del testo, attraverso apposite note a piè di pagina. Pertanto, ho preferito delineare a questo punto una essenziale mappa ragionata di riferimenti, che ripercorre progressivamente alcuni passi del testo. Mappa utile in primo luogo a me (ma spero anche a qualche altro) per proseguire le ricerche. I titoli non preceduti dall'indicazione del nome dell'autore si riferiscono a miei contributi.

La retroattività (in primo luogo della legge, ma anche delle sentenze di accoglimento della Corte costituzionale, del mutamento di giurisprudenza, nonché dell'esercizio di diritti potestativi) è stato il primo tema che ho approfondito nella mia carriera di studioso, nel quadro dello studio rivolto ai rapporti tra tali modificazioni della realtà giuridica e un anteriore accertamento giudiziale: L'efficacia del giudicato civile nel tempo, Milano, 1991. Gli autori con cui ho dialogato si desumono dalle note di La nozione di retroattività della legge, indietro. Se dovessi consigliare una sola opera, indicherei R. QUADRI, Dell'applicazione della legge in generale, in Commentario del codice civile a cura di Scialoja e Branca, Bologna-Roma, 1974.

La necessità di distinguere il regime di efficacia nel tempo delle norme processuali da quello delle norme sostanziali si è fatta strada con lentezza: In tema di autonomia e certezza nella disciplina del processo civile, in Foro it., 2006, I, 136; Tempus regit processum. Per un nuovo principio di diritto intertemporale in materia processuale, indietro.

Ho argomentato la tesi della superfluità della rimessione in termini per rimediare alla situazione venutasi a creare con Cass. 9 settembre 2010, n. 19246 in Il mutamento di giurisprudenza costante della Corte di cassazione in materia di interpretazione di norme processuali come ius superveniens irretroattivo, in Foro it. 2010, V, 311. Ho sinteticamente ripreso e precisato questo pensiero nel mio breve intervento all'Incontro di studi Norme processuali e mutamenti di giurisprudenza, svoltosi presso la Corte di cassazione il 24 maggio 2011. In senso favorevole, la Corte di cassazione. In senso contrario, pur con diversità di accenti, tutte e tre le relazioni dei colleghi processualisti: C. PUNZI, Il ruolo della giurisprudenza e i mutamenti di interpretazione di norme processuali; G. RUFFINI, Mutamenti di giurisprudenza sull'interpretazione di norme processuali e 'giusto processo'; G. COSTANTINO, Il principio di affidamento tra fluidità delle regole e certezza del diritto. Queste tre relazioni sono state pubblicate nella Rivista di diritto processuale, 2011, rispettivamente alle p. 1337 ss,, p. 1390 ss., p. 1073 ss. Sono particolarmente grato alla serrata critica di Giuseppe Ruffini, che mi ha fatto 
avvertire l'esigenza di spiegare in modo migliore ciò che intendevo dire nel mio articolo sul Foro italiano. La mia base di partenza resta La rimessione in termini nel processo civile, Milano, 1996.

Nella letteratura italiana, la tesi secondo la quale il precedente giurisprudenziale è fonte del diritto è argomentata con il massimo impegno da A. PIZZORUSSO, Delle fonti del diritto, in Commentario del codice civile Scialoja-Branca, Bologna-Roma, prima ed., 1977, seconda ed. 2011, p. 705 ss. Su questo tema non può mancare il ricordo delle fondamentali opere di G. GORLA: punto di partenza bibliografico è la voce Giurisprudenza, da lui redatta per l'Enciclopedia del Diritto, vol. XIX, Milano, 1970, p. 489 ss. Nel corso degli anni mi sono appoggiato volentieri alle argomentazioni di A. PIZZORUSSO, da ultimo nelle mie relazioni Corti europee e giudicati nazionali, in Atti del XXVII Congresso nazionale dell'Associazione italiana fra gli studiosi del processo civile, Verona, 25-26 Settembre 2009, Bologna, 2011, p. 239-390 e Transnational Litigation and Elements of Fair Trial, General Report to the XIX. World Congress of the International Association of Procedural Law (Heidelberg, 2011), in P. GOTTWALD, B. HESS (eds.), Procedural Justice, Bielefeld, 2014, p. 493- 536. 8

Il timore nell'appoggiarsi a giustificazioni giuridiche che si appellano alla natura delle cose deriva dalla consapevolezza che il diritto è uno strumento "squisitamente artificiale" (S. RODOTÀ, Antropologia dell'homo dignus, in Riv. crit. dir. priv., 2010).

Una dogmatica giuridica al servizio della vita è quella che ho appreso dall'esempio e dall'insegnamento di ANDREA PROTO PISANI, nonché, sempre se dovessi consigliare una sola opera, da L. MENGONI, Ermeneutica e dogmatica giuridica, Milano, 1996.

Il passo di C. SCHMITT sul rapporto tra l'eccezione e la regola è tratto da Politische Theologie. Vier Kapitel zur Lehre von der Souveränität, München-Leipzig, 1922, trad. it. Teologia politica, in Le categorie del 'politico', a cura di G. Miglio e P. Schiera, Bologna, 1972, p. 34 .

Nel parlare di fatti "tiranni", ho rovesciato il discorso di C. SCHMITT, La tirannia dei valori. Riflessioni di un giurista sulla filosofia dei valori, a cura di G. Gurisatti e F. Volpi, Milano, 2008.

Ho cominciato a riflettere con qualche tratto sistematico sulla normatività dei fatti della vita in Quanto sono normativi i fatti della vita: il rapporto amministrativo, in Diritto pubblico, 2009, p. 159. 